\title{
CHIPAT
CHIAPAS
}

XV Congreso Latinoamericano de Patología de Construcción y XVII Congreso de Control de Calidad en la Construcción CONPAT 2019, Vol. 2, PATOLOGÍA DOI: https://doi.org/10.21041/CONPAT2019/V2PAT341

\section{EVALUACIÓN DE MEZCLA TERNARIA DE CEMENTO PORTLAND, CENIZA VOLANTE E HIDRÓXIDO DE CALCIO: RESISTENCIA MECÁNICA Y DURABILIDAD FRENTE AL ATAQUE POR CARBONATACIÓN}

\author{
P. MEZA-TAVARES ${ }^{1}$, E. U. DE-LOS-SANTOS ${ }^{1}$, G. SANTIAGO-HURTADO ${ }^{1}$, \\ V. M. MORENO-LANDEROS ${ }^{1}$, L. M. REYNOSA-MORALES ${ }^{1}$, J. M. MENDOZA-RANGEL ${ }^{2}$. \\ ${ }^{1}$ Facultad de Ingeniería Civil Unidad Torreón, Universidad Autónoma de Coahuila, Carretera Torreón-Matamoros km \\ 7.8, Ej. El Águila, Torreón, Coahuila, México. \\ ${ }^{2}$ Instituto de Ingeniería Civil, Facultad de Ingeniería Civil, Universidad Autónoma de Nuevo León, Ciudad Universitaria, \\ San Nicolás de los Garza, Nuevo León México.
}

\section{RESUMEN}

El presente trabajo muestra el estudio de cinco mezclas de mortero elaboradas a base de cemento portland con una sustitución alta de Ceniza volante y cal hidratada. El conjunto de mezclas se compone de una referencia solo cemento y el resto se realizaron con una sustitución de $50 \%$ en peso de cemento portland por cuatro proporciones distintas de ceniza volante tipo F e Hidróxido de calcio.

Dichas mezclas fueron elaboradas y evaluadas por medio de pruebas de compresión, deterioro acelerado por carbonatación, porosidad al agua y resistividad eléctrica. La resistencia a compresión se vio disminuida hasta en un $42 \%$; sin embargo, el uso de la cal ayudó a disminuir la profundidad de carbonatación en mezclas con cal respecto a la mezcla donde se utilizó solo ceniza volante. 


\section{INTRODUCCIÓN}

El cambio climático causado por los gases de efecto invernadero nos lleva a buscar alternativas desde todos los ámbitos y áreas de producción de bienes y servicios. La construcción y su quehacer contribuyen en gran medida la liberación de dichos gases y aa la liberación de residuos contaminantes generados durante la explotación de recursos minerales y producción de los materiales (Buchanan \& Honey, 1994) como acero polímeros y componentes del concreto principalmente agregados y cemento (Kim, et al. 2016). Así mismo la concentración de $\mathrm{CO}_{2}$ en la atmósfera conlleva a un deterioro acelerado de las estructuras de concreto reforzado (Talukdar, et al. 2012).

El uso de materiales alternativos en materiales de construcción hechos a base de cemento portland es un área de estudio que ha ganado importancia desde hace algunos años de allí que se busquen alternativas enfocadas a la disminución del uso de cementopor lo que desde finales del siglo 20 diversos esfuerzos e investigaciones se han encaminado a la utilización de materiales de desecho de otras industrias como la acera (escoria granulada de alto horno) (Özbay, et al. 2016), electrónica(microsílica) (Massana, et al. 2018), azucarera (ceniza de bazo de caña) (Bahurudeen, et al. 2015; Arenas-Piedrahita, et al. 2016) y de generación de energía eléctrica (ceniza volante) entre otros. Dentro de los cuales se pueden encontrar estudios con altos niveles de remplazo de ceniza volante (Siddique, 2004; Bouzoubaa \& Lachemi, 2001; Hemalatha \& Ramaswamy, 2017).

Así mismo, se han buscado alternativas a materiales de menor consumo energético para su producción como lo es el uso de polvos de caliza (Wang, 2018) y cal (Gunasekara, et al. 2018), entre los que destaca una ligera mejora en propiedades mecánicas pero sin permitir altos niveles de reemplazo, debido a esto, no se ha logrado analizar las ventajas que ofrecen estos materiales respecto a los materiales alternativos ya mencionados anteriormente.

La investigación presentada en este trabajo busca utilizar una sustitución de cemento portland ordinario 40 (CPO 40) en un 50\% utilizando en su lugar ceniza volante tipo F complementada con Hidróxido de calcio (Cal hidratada) utilizada en la construcción cuyo costo es más económico que el CPO 40 y de fácil adquisición. Por medio de distintos ensayes se busca evaluar la capacidad de la cal hidratada para activar la Ceniza volante y mejorar su desempeño en relación con la alcalinidad que la misma aportará a la mezcla y a la solución de poro.

\section{METODOLOGÍA EXPERIMENTAL}

\subsection{Materiales:}

Para la elaboración de los morteros se utilizaron materiales disponibles localmente en la región, los cuales se describirán brevemente a continuación:

Como cementantes se utilizó un Cemento Portland Ordinario 40 (CPO 40) que cumple con la especificación NMX-C-ONNCCE 414, como materiales cementantes suplementarios se utilizó una hidratada producida por una empresa local que cumple con la norma NMX-C-ONNCCE 005 y Ceniza Volante tipo F (CV) clasificada así por medio de la norma ASTM C 618 y la cual es un residuo generado por la industria carboeléctrica en una planta al norte del estado de Coahuila.

El agregado utilizado para la elaboración de las mezclas fue fino extraído de piedra caliza triturada con tamaño máximo de $6 \mathrm{~mm}$. Adicionalmente se utilizó un aditivo reductor agua a base de poli carboxilatos para mejorar la calidad del mezclado.

\subsection{Mezclas:}

La dosificación de los morteros se realizó en apego a las proporciones más comúnmente utilizadas. El proporcionamiento y nomenclatura las distintas mezclas se describe a continuación: 
- REF es un mortero con una relación 3:1:0.5 de arena, cemento y agua respectivamente para mantener una relación agua/cementante de 0.5 .

- CV: En esta mezcla se sustituyó un 50\% de cemento por ceniza volante en peso basado en las proporciones de la mezcla REF.

- CVCAL-5, CVCAL-10, CVCAL-15: Para estas mezclas se realizó una sustitución en peso del $50 \%$ por ceniza volante y cal, donde parte de la ceniza fue sustituida por cal en $5 \%, 10 \%$ y $15 \%$ (Porcentajes con respecto al 50\% del material total cementante).

Para todas las mezclas se utilizó aditivo reductor de agua a base de carboxilatos con una dosificación de $4 \mathrm{~g} / \mathrm{kg}$ de material cementante. Una vez elaboradas las mezclas los especímenes fueron desmoldados después de 24 horas y se llevaron a curado estándar por 14 días para promover la mejora de sus propiedades, cumplido el periodo de curado se mantuvieron hasta los 28 días de fabricación en ambiente de laboratorio para después realizar los procedimientos que se describen a continuación.

\subsection{Métodos de evaluación:}

Los métodos de evaluación utilizados se describen a continuación:

- Resistencia a compresión en cubos de 100 mm de lado de acuerdo con el estándar NMX-C-ONNCCE 155.

- Resistividad eléctrica por método directo aplicando 30 voltios de corriente directa a especímenes cúbicos de $100 \mathrm{~mm}$ por lado cuyo circuito era alimentado con una fuente de poder por un periodo de 30 segundos para después tomar la lectura de la corriente en miliamperes con ayuda de un multímetro.

- Porosidad y absorción en estado endurecido acorde con la norma ASTM C 642.

- Carbonatación de especímenes cilíndricos de $50 \mathrm{~mm}$ de diámetro. Para esta prueba, los especímenes fueron colocados en desecadores de cristal en los cuales se suministro $\mathrm{CO}_{2}$ de manera constante manteniendo una concentración promedio de $950 \mathrm{ppm}$, temperatura de $23^{\circ} \mathrm{C}$ humedad relativa del $87 \%$, datos tomados por medio de un equipo digital que se mantuvo dentro de los desecadores durante el curso de la experimentación. Al final de la exposición se cortaron rodajas transversales y se rociaron con fenolftaleína para medir la profundidad de mortero carbonatada.

\section{RESULTADOS}

La figura 1 muestra los resultados obtenidos mediante resistencia a compresión de los cubos de mortero. Se puede observar que la resistencia a compresión disminuye significativamente por la sustitución parcial de CPO por CV, sin embargo, las resistencias obtenidas pueden ser equiparables con concretos de buena calidad, para lo cual sería necesario disminuir aún más la relación agua/cementante.

De las mezclas en las que se utilizó cal como sustituto parcial de la ceniza volante no se representa una pérdida significativa de resistencia a compresión respecto a la $\mathrm{CV}$ incluso se observa un descenso proporcional relacionado con el nivel se sustitución de esta. La resistencia obtenida para para la mezcla de referencia a 91 días fue de 52.73 MMa. Mientras que para CV fue $37.63 \mathrm{MPa}$ lo que representa una disminución de casi $29 \%$ llegando hasta el 34\% para CVCAL-15. Mientras que a los 28 días la pérdida de resistencia fue del $35 \%$ al $42 \%$ disminuyendo así la pérdida conforme la maduración de las mezclas, como se puede observar en la literatura el incremento de la resistencia a largo plazo es típico de mezclas con sustituciones cementantes. Por lo que es conveniente un estudio a mayor plazo de la resistencia a compresión para observar su desempeño. 


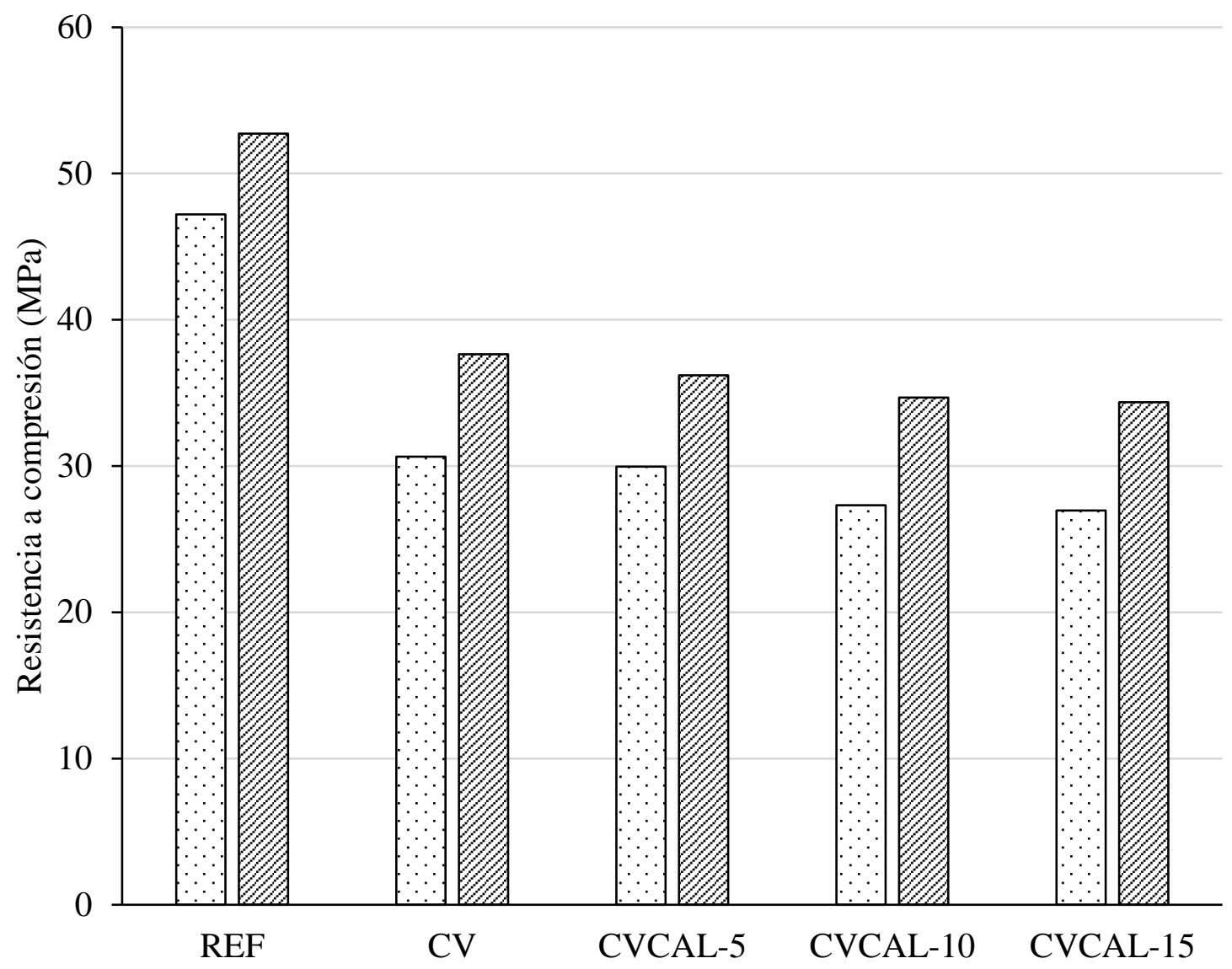

$\square 28$ días $\square 91$ días

Figura 1. Resistencia a compresión de morteros a 28 y 91 días.

La figura 2 muestra la evolución de la resistividad eléctrica, durante la experimentación en la cual podemos observar que la sustitución de CV volante incrementa significativamente la resistividad a largo plazo, es conveniente mencionar que las muestras se curaron y se mantuvieron en ambiente de laboratorio durante el curso de esta medición y la resistividad presenta una disminución en relación con el nivel de cal remplazada. Aunque puede considerarse como un parámetro adverso a la durabilidad esto no sucede para la carbonatación mostrada en la figura 3 donde el uso de cal permite disminuir la profundidad de carbonatación medida a los 184 días de exposición acelerada reduciéndola hasta en poco más de $3 \mathrm{~mm}$ a dicha edad lo que permite establecer que la sustitución de cal nos permite incrementar la durabilidad de mezclas con altas sustituciones de $\mathrm{CV}$ en ambientes con presencia de $\mathrm{CO}_{2}$ representando una ventaja económica. Posiblemente parte de la cal se mantiene en la solución de poro y queda disponible para hacer contrarrestar el ingreso de $\mathrm{CO}_{2}$ manteniendo los niveles de alcalinidad por tiempos más prolongados y que a su vez permite la transmisión de corriente por la disponibilidad de iones de calcio en la solución de poro. 


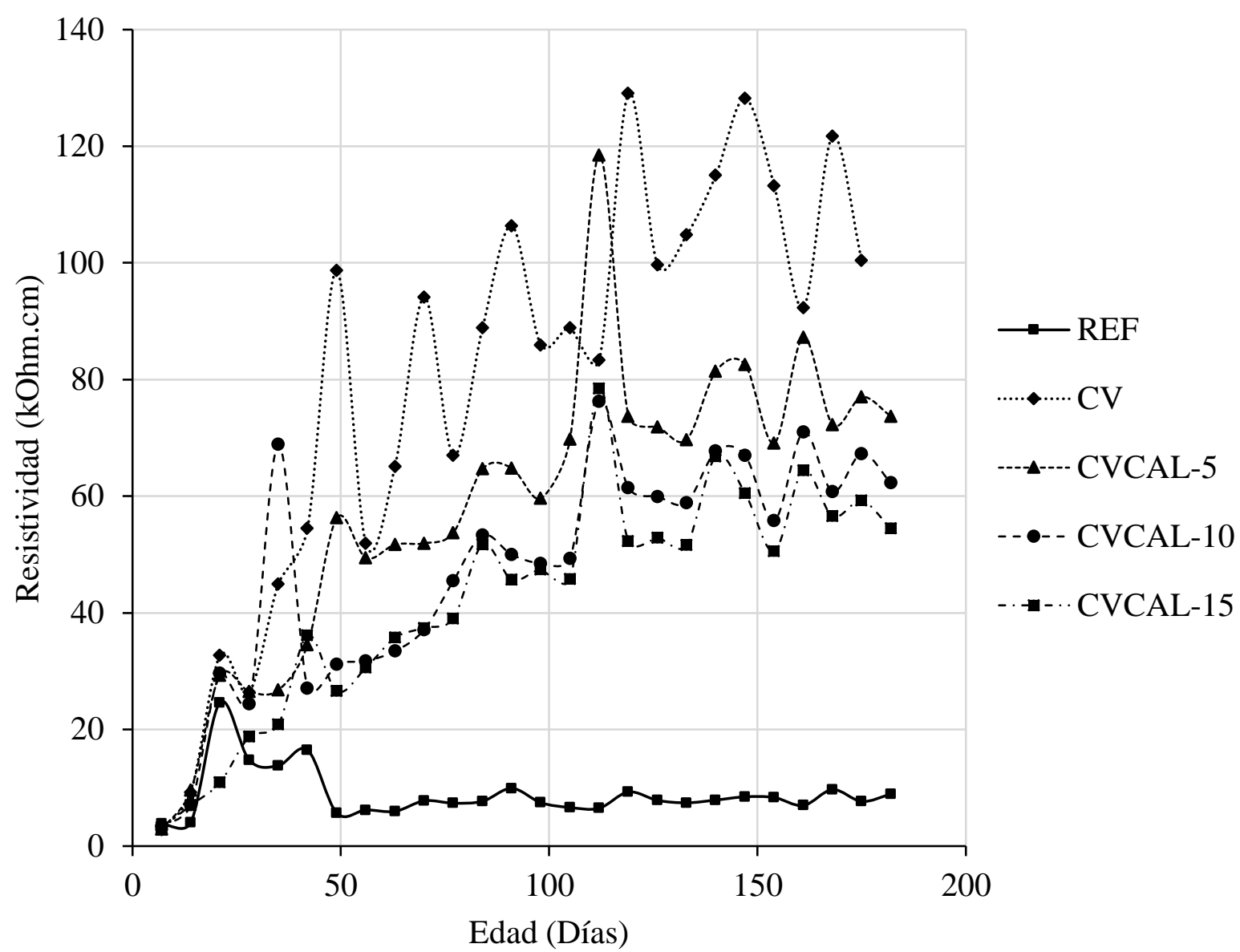

Figura 2. Resistividad eléctrica en etapa de curado y ambiente de laboratorio.

Por otra parte, el uso de ceniza volante y cal no modifica sustancialmente la porosidad de los morteros a 28 días, edad en la cual aún no se ve el efecto de la utilización de la cal. Sin embargo, aunque físicamente no disminuye la porosidad si podemos observar que la principal ventaja del uso de la cal es la función que realiza como barrera química disminuyendo la profundidad de carbonatación, de esta manera el uso de cal resulta benéfico cuando se realizan sustituciones altas de CV.

Tabla 1. Porosidad y absorción de mezclas de mortero a 28 días.

\begin{tabular}{|c|c|c|}
\hline MEZCLA & Porosidad (\%) & Absorción (\%) \\
\hline REF & 20.35 & 9.73 \\
\hline CV & 23.34 & 12.04 \\
\hline CVCAL-5 & 22.72 & 11.84 \\
\hline CVCAL-10 & 21.27 & 11.02 \\
\hline CVCAL-15 & 23.72 & 12.35 \\
\hline
\end{tabular}

La carbonatación en la mezcla REF no se muestra en la figura 3 debido a que hasta el momento de ensayo no presentó signos de avance de carbonatación apreciables. 


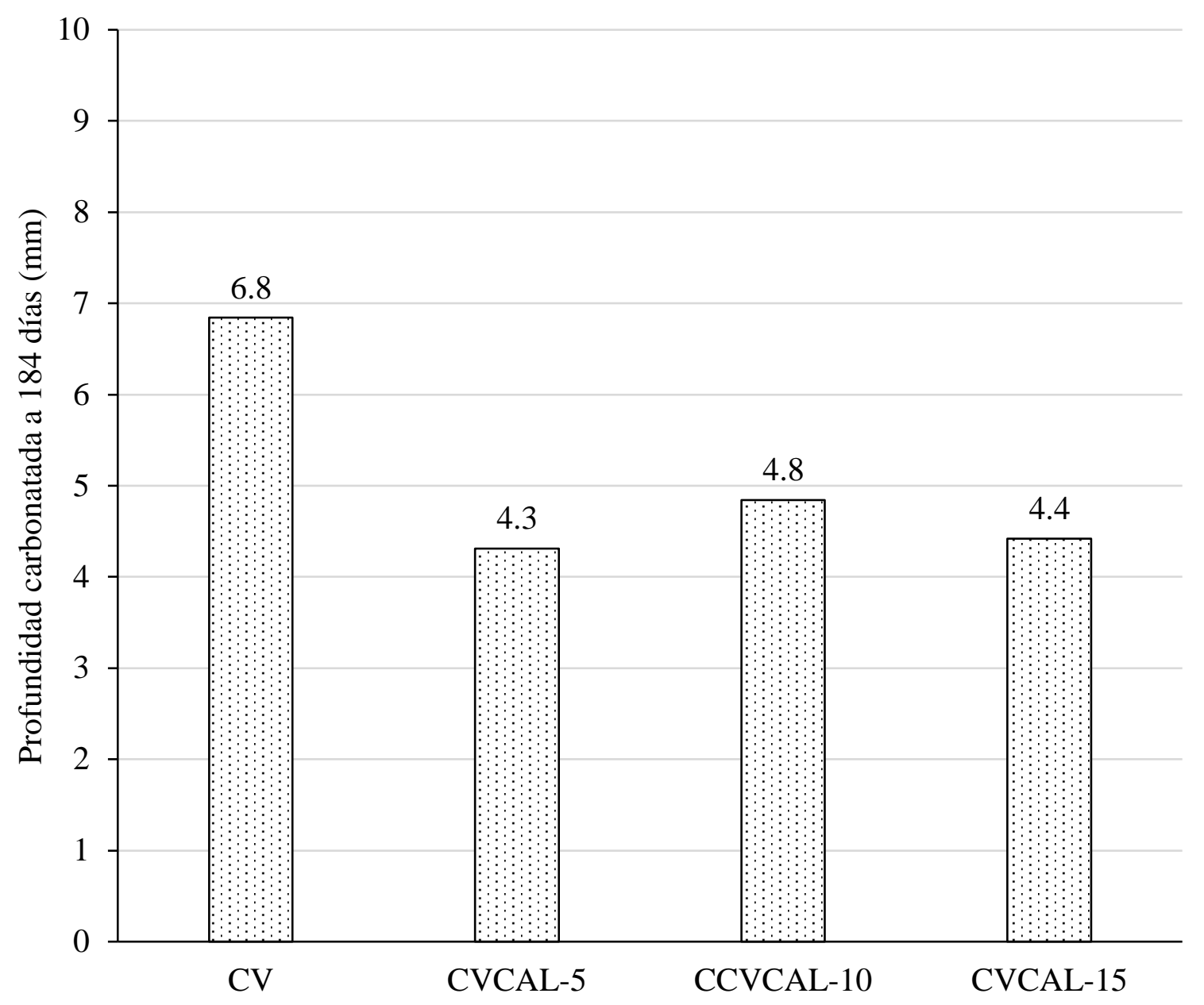

Figura 2. Profundidad de carbonatación de mezclas en deterioro acelerado por $\mathrm{CO}_{2}$.

A pesar de la similitud en porosidad y absorción a 28 días, el comportamiento de la difusión de $\mathrm{CO} 2$ se ven ampliamente influenciada por el uso de la cal lo que representa una solución económa y de alto impacto en la sustentabilidad de esta clase de materiales.

\section{CONCLUSIONES}

Aunque el uso de cementantes suplementarios es un tema que aún no termina por extenderse de manera generalizada en México, esta investigación sugiere una alternativa sustentable y económica para producir morteros con buen desempeño frente al deterioro por carbonatación.

El uso de ceniza volante en altos niveles permite que parte de la ceniza quede sin reaccionar, sin embargo, el uso de cal hidratada puede ayudar a incrementa la durabilidad utilizando sustituciones de cal para mejora de dichas mezclas.

Aunque la resistencia s compresión disminuye significativamente por el uso de $50 \%$ de ceniza volante en sustitución, se observa una disminución en la perdida a 91 días con respecto a REF para todas las mezclas de CV y Cal.

Es importante la investigación a largo plazo de este tipo de mezclas para evaluar sus propiedades, desde el aspecto de los efectos que pueda aportar la cal a la CV como mejora de resistencia mecánica y porosidad. 


\section{AGRADECIMIENTOS.}

El autor desea expresar su agradecimiento al Consejo Nacional de Ciencia y Tecnología (CONACyT) por la beca otorgada para el curso del posgrado durante el cual se llevó a cabo está investigación.

\section{BIBLIOGRAFÍA}

Buchanan, A. H., \& Honey, B. G. (1994), Energy and carbon dioxide implications of building construction, Energy and Buildings, Volume 20, Issue 3, 1994, Pages 205-217. https://doi.org/10.1016/0378-7788(94)90024-8

Kim, T., Chae, C., Kim, G., \& Jang, H. (2016), Analysis of $\mathrm{CO}_{2}$ emission characteristics of concrete used at construction sites. Sustainability 2016, 8(4), 348; https://doi.org/10.3390/su8040348

Talukdar, S., Banthia, N., \& Grace, J. R. (2012), Carbonation in concrete infrastructure in the context of global climate change-Part 1: Experimental results and model development. Cement and Concrete Composites, Vol. 34 (8), págs. 924-930. https://doi.org/10.1016/j.cemconcomp.2012.04.011

Özbay, E., Erdemir, M., \& Durmuş, H. İ. (2016), Utilization and efficiency of ground granulated blast furnace slag on concrete properties-A review. Construction and Bulding Materials, Vol. 105. https://doi.org/10.1016/j.conbuildmat.2015.12.153

Massana, J., Reyes, E., Bernal, J., León, N., \& Sánchez-Espinosa, E. (2018), Influence of nano-and micro-silica additions on the durability of a high-performance self-compacting concrete. Construction and Building Materials, Vol. 165, págs. 93-103. https://doi.org/10.1016/j.conbuildmat.2017.12.100

Bahurudeen, A., Kanraj, D., Dev, V. G., \& Santhanam, M. (2015), Performance evaluation of sugarcane bagasse ash blended cement in concrete. Cement and Concrete Composites, Vol. 59, págs. 77-88. https://doi.org/10.1016/j.cemconcomp.2015.03.004

Arenas-Piedrahita, J. C., Montes-García, P., Mendoza-Rangel, J. M., Calvo, H. L., Valdez-Tamez, P. L., \& Martínez-Reyes, J. (2016), Mechanical and durability properties of mortars prepared with untreated sugarcane bagasse ash and untreated fly ash. Construction and building materials, Vol. 105, págs. 69-81. https://doi.org/10.1016/j.conbuildmat.2015.12.047

Siddique, R. 2004, Performance characteristics of high-volume Class $F$ fly ash concrete. Cement and Concrete Research, Vol. 34 (3), págs. 487-493. https://doi.org/10.1016/j.cemconres.2003.09.002

Bouzoubaâ, N., \& Lachemi, M. (2001), Self-compacting concrete incorporating high volumes of class F fly ash: Preliminary results. Vol. 31 (3), págs. 413-420. https://doi.org/10.1016/S00088846(00)00504-4

Hemalatha, T., \& Ramaswamy, A. (2017), A review on fly ash characteristics-Towards promoting high volume utilization in developing sustainable concrete. Journal of cleaner production, Vol. 147, págs. 546-559. https://doi.org/10.1016/j.jclepro.2017.01.114

Wang, X. Y. (2018). Analysis of hydration and strength optimization of cement-fly ash-limestone ternary blended concrete. Construction and Building Materials. Vol. 166, págs. 130-140. https://doi.org/10.1016/j.conbuildmat.2018.01.058

Gunasekara, C., Law, D.W., \& Setunge, S. (2018). Design of Ternary Blend High-Volume Fly Ash Concrete Mixes using Hydrated Lime. ICDCS 2018. 